In this presentation it is argued that: (i) comprehensive sexuality education needs to start in early childhood and be built on in primary and secondary schooling; (ii) comprehensive sexuality education needs to be collaboratively informed by sexuality educators and health professionals; (iii) sexuality education programs in school need to be developed in partnership with parents and include parental education on best practices around talking with their children about sexual knowledge and relationships; and (iv) comprehensive sexuality education is critical to building young people's sexuality literacy, respect for gender and sexuality difference, and awareness of sexual ethics, which are all central to children and young people's health and well-being and the development of their sexual citizenship.

A mixed-methods approach was employed across the major research informing this presentation. The ARC Discovery funded project included an exploration of primary school children's understandings of respect, sexual knowledge and relationships and the origins of this information, as well as parents' and educators' perspectives and practices talking with children about sexuality education. Data were gathered through surveys, focus groups and individual interviews with parents and educators, and focus groups and interviews with children. The CRC funded project, Growing Up Queer, included a validated online survey of LGBTQI Young People, aged 16-27 years, completed by 1,032 participants nationally. Two focus groups were also conducted, one with LGBTIQ young people, and the other with staff from a support service for gender and sexuality diverse young people.

\section{SO2.4 ENGAGING BOYS AND MEN AS ACTORS IN VIOLENCE PREVENTION}

Bonney Corbin*. True Relationships and Reproductive Health, (formerly Family Planning Queensland), Australia

\subsection{6/sextrans-2015-052270.25}

Popular culture surrounding sexual and gender based violence stereotypes individual actors and their intimate relationships. These stereotypes limit opportunities for conflict transformation pedagogy. As feminism returns to vogue amongst youth populations and concepts of gender diversity enter mainstream school discussion, students and their school communities are calling for alternative approaches to violence prevention education. Doing so successfully, requires moving beyond education about personal standpoints and attitudes to consider the relationships of power enabled via informal and formal community structures including via online and offline media. Considering violence prevention education holistically, every individual within a school community has opportunities to provide violence prevention education. This presentation will provide an overview of core concepts of violence prevention theory including masculinities, continuum models and structural analysis. Demonstrating how theory can be translated in practice, these concepts will be explained alongside practice-based examples from international work to prevent sexual and gender based violence.

\section{S02.5 THE POLITICS OF PLEASURE IN SEXUALITY EDUCATION: YOUNG PEOPLE'S PERSPECTIVES}

Louisa Allen*. University of Auckland, Auckland, New Zealand
The inclusion of pleasure and desire have been important in the vision for sexuality education for the past 20 years. This paper continues this conversation by exploring young people's interest and ideas about incorporating pleasure within sexuality education at school. Drawing on data from focus group and survey methods young people highlight some of the challenges facing this topic as a curricula component. Participants felt sexual pleasure was relevant to their lives and displayed a significant interest in receiving this information provided it was delivered in a particular format. Responses provide insights into some of the politics which surround the inclusion of pleasure in sexuality education. Taking into account young people's perspectives this paper encourages an acknowledgement and interrogation of these politics and their implications for what gets 'taught' as pleasure in sexuality programmes.

\section{S03 - Global and regional estimates of prevalent and incident STI}

\section{S03.1 GLOBAL AND REGIONAL ESTIMATES OF CHLAMYDIA, GONORRHOEA, TRICHOMONIASIS AND SYPHILIS IN 2012}

Sami Gottlieb*. World Health Organization, Geneva, Switzerland

\subsection{6/sextrans-2015-052270.27}

Background The World Health Organization (WHO) periodically estimates global and regional prevalence and incidence of four curable sexually transmitted infections (STIs): chlamydia, gonorrhoea, trichomoniasis and syphilis.

Methods For chlamydia, gonorrhoea, and trichomoniasis, WHO estimates for 2012 were based upon literature reviews of prevalence data from 2005 through 2012 among general populations. For syphilis, nationally reported data on syphilis seroprevalence among antenatal care attendees were used. Data were adjusted for laboratory test type, geography, age, and high risk subpopulations, and combined using a Bayesian meta-analytic approach. Regional incidence estimates were generated from prevalence estimates by adjusting for average duration of infection.

Results In 2012 the estimated global prevalence among women aged 15-49 years of chlamydia was 4.2\% (95\% uncertainty interval (UI): $3.7-4.7 \%)$, gonorrhoea $0.8 \%(0.6-1.0 \%)$, trichomoniasis $5.0 \%$ (4.0-6.4\%), and syphilis $0.5 \% \quad(0.4-0.6 \%)$; among men, estimated chlamydia prevalence was $2.7 \%(2.0-$ $3.6 \%)$, gonorrhoea $0.6 \%(0.4-0.9 \%)$, trichomoniasis $0.6 \%(0.4-$ $0.8 \%)$, and syphilis $0.48 \%(0.3-0.7 \%)$. These figures correspond to an estimated 131 million new cases of chlamydia (100-166 million), 78 million of gonorrhoea (53-110 million), 143 million of trichomoniasis (98-202 million), and 6 million of syphilis (48 million) in 2012. Prevalence and incidence estimates varied by region and sex.

Conclusions Estimated global prevalence and incidence of chlamydia, gonorrhoea, trichomoniasis, and syphilis in adult women and men remain high, with nearly one million new infections each day. These estimates highlight the urgent need for well-recognized effective interventions for STI prevention, screening, diagnosis, and treatment to be made more widely available. Improved estimation methods are needed to allow use of more varied data and to generate national-level estimates. 